\title{
Ein Jahr mit Waisenkindern teilen
}

\section{Freiwilligendienst in den Kinderdörfern von nuestros pequeños hermanos}

DAGMAR SCHNEIDER

Dagmar Schneider leitet die Presse und Öffentlichkeitsarbeit von nuestros pequeños hermanos (nph) deutschland e. V.

dagmar.schneider@nph-deutschland.org

\author{
Das christliche Kinderhilfswerk nuestros \\ pequeños hermanos bietet in Lateinamerika \\ verwaisten oder verlassenen Kindern eine \\ Zufluchtstätte und Ausbildungsmöglichkeiten. \\ Die örtlichen Teams werden in ihrer Arbeit \\ unterstützt von Freiwilligen aus Deutschland.
}

Eine Momentaufnahme: Franco strahlt und streckt seine Arme aus: »Hola! Vamos a jugar? «, fragt der kleine Peruaner seine Betreuerin und schaut sie erwartungsvoll an. Spielen ist die Lieblingsbeschäftigung des Dreijährigen und zusammen mit Anne Scherer verbringt er Stunden im Sandkasten. Dort backt er Kuchen, baut Türme und gräbt Löcher.

Anne Scherer ist eine von 15 jungen Frauen und Männern aus Deutschland, die derzeit Freiwilligendienst in einem der Kinderdörfer von nuestros pequeños hermanos (spanisch für »Unsere kleinen Brüder und Schwestern«) leisten. Das internationale Kinderhilfswerk nuestros pequeños hermanos (nph) ist in Mexiko, Honduras, Haiti, Nicaragua, Guatemala, El Salvador, der Dominikanischen Republik, Peru und Bolivien aktiv.

Dort kümmern sich die Mitarbeiter und Freiwilligen um Waisenkinder und um Mädchen und Jungen, die von ihren Eltern verlassen, misshandelt oder missbraucht wurden. nph hat in Lateinamerika Kinderdörfer, Schulen, Ausbildungswerkstätten sowie medizinische und therapeutische Einrichtungen aufgebaut. 3.500 Mädchen und Jungen haben in den Heimen ein neues, liebevolles Zuhause gefunden, weitere 250.000 notleidende Menschen erhalten humanitäre Hilfe. nuestros pequeños hermanos versteht sich als eine Familie, zu der neben den Kindern und Angestellten auch die Freiwilligen zählen. In den Kinderdörfern nehmen sie eine wichtige Rolle ein: Sie arbeiten Hand in Hand mit den Mitarbeitern vor Ort und unterstützen sie durch ihr Wissen und Engagement.

Oft besitzen die Freiwilligen Qualifikationen, die es in vielen Ländern in Lateinamerika nicht gibt oder nur in unzureichender Anzahl. Corinna Lawrenz beispielsweise leistete als Ärztin ein Freiwilligenjahr im Kinderdorf in Guatemala. Dort kümmerte sie sich um kranke Kinder und Jugendliche, führte Vorsorgeuntersuchungen durch und half, die medizinischen Standards weiter $\mathrm{zu}$ verbessern. Inzwischen arbeitet die 29-Jährige im internationalen medizinischen Team von nph mit und reist mehrfach im Jahr ins Kinderdorf nach Mexiko.

Neben medizinischem Fachpersonal sind in den Kinderdörfern auch Therapeuten dringend notwendig. Denn viele der Mädchen und Jungen haben, bevor sie ins Kinderdorf kamen, schwere körperliche und seelische Wunden davongetragen. Sie sind traumatisiert und brauchen therapeutische Hilfe. Psychologen, Physio-, Ergo- und Kunsttherapeuten kümmern sich um 
diese Kinder, so dass die Wunden mit der Zeit heilen können. Oft verschwinden dann auch die Verhaltensauffälligkeiten, die Folgen der körperlichen und seelischen Misshandlungen sind. Da nph eigene Kindergärten, Schulen und Ausbildungsprogramme betreibt, sind Pädagogen im Kinderdorf willkommen. Durch eine gute Schulbildung und eine fundierte Ausbildung eröffnen die Erzieher und Lehrer den Kindern eine Chance auf ein menschenwürdiges und selbstbestimmtes Leben, fernab von Not und Armut.

Neben den Fachkräften können bei nph auch junge Menschen Freiwilligendienst leisten, die keine spezifische Berufsausbildung haben. Sie arbeiten im Kinderdorf in der Kinderbetreuung, als Nachhilfelehrer oder in der Koordination von Freizeitaktivitäten. Für die Mädchen und Jungen in den Kinderdörfern sind die Freiwilligen wichtige Bezugspersonen: Mit ihnen können sie Konflikte besprechen oder einfach nur kuscheln. Wenn das Dienstjahr zu Ende geht, sind viele Kinder traurig. Sie wissen aber auch, dass viele Freiwillige den Kontakt halten und eines Tages vielleicht sogar in »ihre "Kinderdörfer zurückkehren und ihre Schützlinge besuchen.

\section{Voraussetzungen für den Freiwilligendienst}

Viele jungen Menschen in Deutschland träumen davon fremde Länder und Kulturen kennenzulernen. Manche von ihnen verwirklichen ihren Traum und reisen durch die Welt. Andere wiederum verbinden ihre Neugierde auf andere Kulturkreise mit sozialem Engagement.

Eine davon ist Lilith von Becker. Die 22-Jährige wollte mehr von der Welt kennenlernen und einen Beitrag dazu leisten, dass Kinder in Lateinamerika ein besseres Leben und vor allem eine Zukunft erhalten. Im letzten Jahr lebte und arbeitete sie als Ergotherapeutin mit den Kindern von nph in Peru. Bevor Becker ihren Dienst antrat, musste sie zunächst Spanisch lernen. Sprachkenntnisse sind für einen Freiwilligendienst bei nph ein Muss. Denn die Organisa-

\section{Förderprogramme erwünscht}

nph deutschland ist anerkannter Träger für die Förderprogramme Internationaler Jugendfreiwilligendienst (IJFD) und »weltwärts«. Der Internationale

"Für die Mädchen und Jungen in den

\section{Kinderdörfern sind die Freiwilligen aus Deutschland wichtige Bezugspersonen«}

tion ist ausschließlich in Lateinameri$\mathrm{ka}$ aktiv und Spanisch ist wichtig für den Austausch mit den Kindern und Absprachen mit den einheimischen Mitarbeitern. Eine Ausnahme bildet Haiti. Dort engagiert sich nph seit 25 Jahren für die notleidenden Menschen. Die Landessprachen in Haiti sind Kreol und Französisch.

Freiwillige wie Lilith von Becker, Corinna Lawrenz und Anne Scherer haben sich bereiterklärt, mindestens ein Jahr ihres Lebens mit den Mädchen und Jungen von nph zu teilen. Kurzzeiteinsätze sind bei nph nur bei dringend benötigten Fachkräften und Senior Experten möglich. Weil die Arbeit mit den Kindern eine gewisse Reife und Geduld erfordert, liegt das Mindestalter für einen Freiwilligendienst bei nph bei 21 Jahren. Nur in Ausnahmefällen akzeptiert die Organisation auch jüngere Bewerber. Die Kinderdörfer kommen für die Unterkunft und Verpflegung der Freiwilligen auf und zahlen ihnen ein kleines Taschengeld. Die Reisekosten zum Einsatzort und zurück muss der Freiwillige selbst tragen, wenn diese nicht durch staatliche Programme gefördert werden.
Jugendfreiwilligendienst richtet sich an Jugendliche im Alter von 18 bis 26 Jahren. Er wird vom Bundesministerium für Familie, Senioren, Frauen und Jugend gefördert. Der Dienst kann weltweit in sozialen Einrichtungen geleistet werden (Informationen: www.internationaler-jugend-freiwilligendienst.de).

»weltwärts" ist ein entwicklungspolitischer Freiwilligendienst. Das Programm wurde 2008 vom Bundesministerium für wirtschaftliche $\mathrm{Zu}$ sammenarbeit und Entwicklung ins Leben gerufen. Es richtet sich an junge Menschen im Alter zwischen 18 und 28 Jahren und ist als Lern- und Hilfsdienst konzipiert. Freiwillige, die im Rahmen dieses Programms einen Dienst leisten, bekommen einen Teil ihrer persönlichen Kosten erstattet. Für »weltwärts«-Freiwillige gelten ähnliche Rahmenbedingungen wie für andere Freiwilligen, die bei nph ihren Dienst leisten. Der Einsatz beginnt entweder im Januar oder im Juli eines Jahres (Informationen: www. weltwaerts.de).

Für kürzere Einsätze arbeitet nph mit dem Senior Experten Service, Bonn, zusammen. Der Senior Experten Service vermittelt tatkräftige und hoch qualifizierte Senioren zu Einsätzen. Für Organisationen wie nph ist dieses Expertenwissen von unschätzbarem Wert.

\section{Qualität}

Damit der Freiwilligeneinsatz ein Erfolg für alle Beteiligten wird, hat nph sich dem fid-Qualitätsmanagement verpflichtet. Als Grundlage dient der fid-Qualitätsmanagement-Kriterienkatalog. Alle Bewerber durchlaufen ein internationales Bewerbungsverfahren, beginnend mit einem Fragebogen, der nph zur Vorauswahl dient. Er vermit- 
telt einen ersten Eindruck des Bewerbers. Beim Informationstag, zu dem die Bewerber nach Karlsruhe eingeladen werden, lernen sich die Bewerber und die Mitarbeiter von nph deutschland persönlich kennen. Während der Gespräche loten beide Parteien die gegenseitigen Erwartungen aus und bauen Vertrauen auf. Der Fokus beim Informationstag liegt aber auf den vielfältigen Herausforderungen des Freiwilligenjahres, von der Unterbringung über den Kulturschock bis hin zu Geschlechterfragen.

Die Entscheidung über eine $\mathrm{Zu}$ - und Absage liegt bei den Verantwortlichen in den Kinderdörfern. Sie stellen sich aus einer Vielzahl an internationalen Bewerbungen ihr Freiwilligenteam zusammen. Dieser Schritt ist wichtig, denn nur wenn die Heime selbst entscheiden, ist garantiert, dass die Verantwortlichen vor Ort die Freiwilligen akzeptieren und ausreichend unterstützen.

Sobald die Freiwilligen eine Zusage erhalten, starten sie in die Phase der Vorbereitung, die für einen positiven Verlauf des Einsatzes bedeutsam ist. Zur Vorbereitung besuchen die künftigen Freiwilligen drei Seminare:

- Die erste Veranstaltung ist ein zehntägiger Vorbereitungskurs bei der fid-Service- und Beratungsstelle für internationale Freiwilligendienste in der Arbeitsgemeinschaft für Entwicklungshilfe in Köln. Hier werden die künftigen Freiwilligen auf die Herausforderungen vorbereitet, die sie in ihren Gastländern erwarten. Gemeinsam mit den Seminarleitern erschließen sie internationale soziale Fragen. Kulturelle und gesellschaftliche Besonderheiten des Gastlandes liegen dabei ebenso im Fokus, wie die Konfrontation mit Armut, Elend und Tod.

- Das zweite Seminar, der nph-Vorbereitungsworkshop, ist ein zweitägiger Kurs in Baden-Baden. Er wird von ehemaligen Freiwilligen geleitet. Sie berichten von den Kindern, ihren eigenen Erfahrungen und den $\mathrm{He}$ rausforderungen, vor die sie während ihres Dienstes gestellt waren. Durch ihre Schilderungen sensibilisieren sie die künftigen Freiwilligen und bereiten sie auf die Aufgaben im Kinderdorf vor. Im Dialog zwischen den ehemaligen und künftigen
Freiwilligen klären sich viele offene Fragen. Weil die "alten Hasen« aus der Praxis berichten, können sie den künftigen Freiwilligen viele wertvolle Tipps an die Hand geben, die ihnen das Leben in Lateinamerika und im Kinderdorf erleichtern.

- Der dritte Kurs, den die künftigen Freiwilligen absolvieren, ist ein zweitägiges Vorbereitungsseminar bei nph deutschland in Karlsruhe. Das Seminar leiten die Mitarbeiter des Karlsruher Büros, die für die Freiwilligen das Bindeglied zwischen Deutschland und Lateinamerika sein werden. Die Freiwilligenkoordinatoren führen die kurz vor der Ausreise Stehenden in die Organisations- und Kommunikationsstrukturen in den Kinderdörfern ein. Sie thematisieren mögliche Konflikte und wie der Freiwillige im Kinderdorf diese Konflikte lösen kann. Das Seminar lässt Raum, um noch offene Fragen zu klären. Bei nph deutschland ist die Teilnahme an allen drei Veranstaltungen Pflicht, weil der Organisation sehr daran gelegen ist, die Freiwilligen möglichst gut vorbereitet in ihr jeweiliges Gastland zu senden.

\section{Begleitung auch über das Dienstende hinaus}

nph deutschland lädt die Freiwilligen nach ihrer Rückkehr nach Deutschland zu einem Gespräch ein. Die Mitarbeiter der Organisation ermutigen die ehemaligen Freiwilligen, von ihren Erfahrungen zu berichten.

Das Treffen dient den zurückgekehrten Freiwilligen auch dazu, sich miteinander auszutauschen. Bei Interesse ermöglicht nph deutschland eine Teilnahme an einem fid-Rückkehrerseminar und bietet die Möglichkeit, sich mit ehemaligen nph-Freiwilligen zu vernetzen und sich weiterhin für $n p h$ zu engagieren.

Viele Freiwillige nehmen diese Angebote dankend an. Sie fühlen sich durch ihren Freiwilligendienst und vor allem die Erfahrungen mit den Kindern sehr eng mit nph verbunden und betrachten sich als Teil der großen nph-Familie.

\section{"Standardwerk"}

Roland Wolf, SAE 1/05, zur Vorauflage

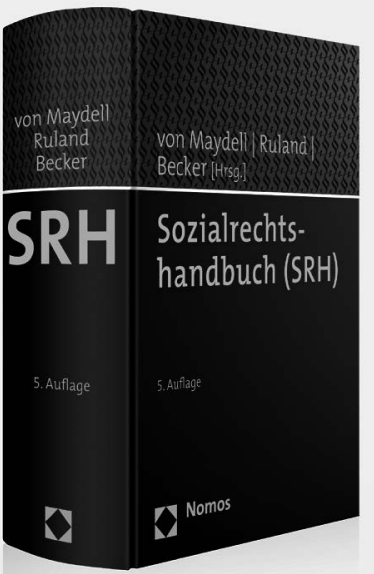

\section{Sozialrechtshandbuch (SRH)}

Herausgegeben von Prof. Dr. Bernd Baron von Maydell, Prof. Dr. Franz Ruland und Prof. Dr. Ulrich Becker, LL.M.

5. Auflage 2012, 1.625 S.,

geb., 158,- $€$

ISBN 978-3-8329-6462-7

\section{Topaktuell}

\section{Mit den neuesten}

Entwicklungen

- Gesundheitsreform

- Rentenversicherung

- Hartz IV-Regelsätze

- Eingliederungschancengesetz

- Europäisches Sozialrecht 
»Tun Sie gelegentlich etwas, womit Sie weniger oder gar nichts verdienen. Es zahlit sich aus."

Oliver Hassencamp, deutscher Schriftsteller (1921-1988)

„So schnell der Hase der freiwilligen Tätigkeit auch
rennt, der lgel Staat war immer schon da."
Ralf Dahrendorf, deutscher Soziologe (1929-2009)

"Auch der Freiwilligste hat seinen Grund."

Albert Wendt, deutscher Schriftsteller (geb. 1948)

"Was früher selbstverständlich war, ist heute eine Dienstleistung."

Tobias Grüterich, deutscher Schriftsteller (geb. 1978)

„In Zeiten politischer Krisen ist es für den ehrenhaften Menschen nicht am schwersten, seine Pflicht zu tun, sondern sie überhaupt zu kennen." Luis de Bonald, französischer Staatsmann und Philosoph (1754-1840)

»Eine Gesellschaft lebt von Flexibilität und Wagnis, von Neugier und Aufbruch. Sie lebt aber auch von Treue und gegenseitigen

Verpflichtungen, von Solidarität, von Engagement und Hingabe. Das taucht in keiner Effizienzrechnung auf, aber davon geht der Wärmestrom aus, von dem wir leben." Johannes Rau, deutscher Politiker (1931-2006)

"Wollen wir vermeiden, dass das Alter zu einer spöttischen Parodie unserer früheren Existenz wird, so gibt es nur eine einzige Lösung, nämlich Ziele zu verfolgen, die unserem Leben einen Sinn verleihen: das hingebungsvolle Tätigsein für einzelne, für Gruppen oder für eine Sache, Sozialarbeit, politische, geistige oder schöpferische Arbeit." Simone de Beauvoir, französische Schriftstellerin (1908-1986) 